(1)

CrossMark

\title{
Targeting harmful use of alcohol for prevention and treatment of tuberculosis: a call for action
}

\author{
Mario Raviglione ${ }^{1}$ and Vladimir Poznyak ${ }^{2}$
}

Affiliations: ${ }^{1}$ Global TB Programme, WHO, Geneva, Switzerland. ${ }^{2}$ Mental Health and Substance Abuse, WHO, Geneva, Switzerland.

Correspondence: Mario Raviglione, Global TB Programme, World Health Organization, 20 Avenue Appia, Geneva-1211, Switzerland. E-mail: raviglionemđawho.int

@ERSpublications

Harmful use of alcohol favors development of tuberculosis and poor outcomes http://ow.ly/ oWKs30cyHcx

Cite this article as: Raviglione M, Poznyak V. Targeting harmful use of alcohol for prevention and treatment of tuberculosis: a call for action. Eur Respir J 2017; 50: 1700946 [https://doi.org/10.1183/ 13993003.00946-2017].

Over the past few years, the increasing role of risk factors and determinants of tuberculosis (TB) has been sufficiently elucidated $[1,2]$. A number of noncommunicable conditions that are associated with TB have been identified [3]. These are particularly important at the start of the new millennium, as more and more low- and middle-income countries have entered a phase of epidemiological transition characterised by growing epidemics of noncommunicable diseases and conditions replacing infectious diseases as the main causes of ill health and deaths $[4,5]$.

The harmful use of alcohol is one of the four key risk factors for major noncommunicable diseases $[6,7]$ that has also emerged as a risk factor for some infectious diseases, including TB $[7,8]$. The WHO Global strategy to reduce the harmful use of alcohol, endorsed by the World Health Assembly in 2010, defines broadly the "harmful use of alcohol" as "drinking that causes detrimental health and social consequences for the drinker, the people around the drinker and society at large, as well as the patterns of drinking that are associated with increased risk of adverse health outcomes" [9].

The links between heavy drinking and TB had been described in the medical literature since the middle of the past century [10], but only recently, due to results of well-designed studies summarised in subsequent systematic reviews and meta-analyses [11, 12], the causal relationship of TB with heavy alcohol consumption and alcohol use disorders had been firmly established and taken into consideration in the estimates of alcohol-attributable disease burden [13-15].

In this context, the article of ImTiaz et al. [16] in the European Respiratory Journal is an important step forward in quantifying the contribution of alcohol consumption to the burden of TB at global and national levels, as well as the contribution of the TB-related morbidity and mortality to the overall alcohol-attributable disease burden. These new estimates suggest that in 2014 about 170000 deaths due to TB were attributable to alcohol consumption worldwide, and as many as $17 \%$ of incident cases of TB and $15 \%$ of deaths due to TB could be prevented by eliminating the harmful use of alcohol. Hence,

Received: May 082017 | Accepted: May 152017

M. Raviglione and V. Poznyak are staff members of the World Health Organization. The authors alone are responsible for the views expressed in this publication and they do not necessarily represent the decisions or policies of the World Health Organization.

Conflict of interest: None declared.

The content of this work is copyright of the authors or their employers. Design and branding are copyright @ERS 2017. 
identification and implementation of effective strategies and interventions to reduce the harmful use of alcohol is of global relevance and significant importance for TB prevention and care. There are several practical implications that follow these findings.

First, to promote public health action one must acknowledge the overall relevance of reducing harmful use of alcohol for TB control. Development, implementation and enforcement of alcohol control policies have a potential to alleviate the role of the harmful use of alcohol among the key risk factors for TB and associated mortality in the population. This is particularly important for high-burden countries suffering from the dual burden of TB epidemics and the harmful use of alcohol.

Second, the implication for action is linked to targeted prevention and treatment interventions focused on high risk groups and individuals, and delivered within health and social care systems. In recent years the role of heavy drinking and alcohol use disorders in the incidence and progression of $\mathrm{TB}$, as well as their impact on TB treatment adherence has been documented in an increasing number of countries in different continents, including India [17], Kazakhstan [18], Poland [19], Vietnam [20], South Africa [21, 22] and Uganda [23]. The role of heavy drinking and alcohol use disorders in favouring development and spread of drug-resistant TB has been confirmed in studies conducted in diverse economic and cultural contexts [24-27]. In spite of increasingly documented and quantified contribution of the harmful use of alcohol to development and progression of TB and poor treatment outcomes, the priority in public health given to addressing this risk factor is still far from what could be expected based on the evidence available. Thus far, very few pilot projects have assessed the feasibility and effectiveness of interventions focused on alcohol use disorders in management of TB [28]; results of integrating alcohol treatment interventions into routine TB care for patients with alcohol dependence in one Russian city are not particularly encouraging [29]. There is an urgent need to invest in identifying the most appropriate and efficient treatment options and delivery models for people affected by TB and alcohol use disorders.

There are many challenges which need still to be addressed. For population-based epidemiological studies it is important to further explore the thresholds of alcohol exposure that have an impact on incidence and progression of TB. The article by ImTiaz et al. [16] indicates alcohol exposure thresholds used in the analysis which are based on currently available evidence and are different for men and women. However, this issue definitely requires further research as the operationalisation of alcohol exposure and the use of compatible, comparable and clinically relevant measures across the studies continues to be a challenge; this hampers translation of research findings into clinical practice and their use in prevention strategies and interventions. For clinicians it is important to identify precisely defined health conditions such as alcohol dependence or alcohol use disorder of specified severity, and preferably discrete patterns of alcohol use, including the hazardous use of alcohol, so that the most effective interventions and treatment options for patients with TB can be designed. Currently a range of treatment options with proven effectiveness is available for management of hazardous drinking and alcohol use disorders from screening and brief interventions for risky patterns of alcohol consumption to pharmacological and structured psychosocial interventions for management of alcohol dependence [30-32].

In spite of current gaps in our knowledge, there are two major public health approaches that are justified in view of all accumulated evidence and new data presented by ImTiaz et al. [16]. The first approach is related to systematic screening for alcohol use and early identification of alcohol use disorders in all patients engaged with prevention and treatment services for TB. Health professionals in these settings should be trained to identify not only alcohol use disorders, but also patterns of alcohol use associated with increased risk of negative health consequences including TB-related risks. In other words, hazardous patterns of alcohol consumption and alcohol use disorders must be recognised and appropriate interventions and treatment options implemented. These may include simple advice and brief interventions or expand to pharmacotherapy of alcohol dependence, if this is diagnosed, and referral to specialists for support in organising and implementing structured treatment.

The second approach is related to active screening and identification of TB cases among people with alcohol use disorders, including those enrolled in special treatment programmes for alcohol dependence. Special attention would need to be paid to high risk groups for these conditions including the homeless, people released from prisons, and those using other psychoactive substances such as tobacco and illicit drugs. Individuals with alcohol and comorbid drug use disorders present a particularly high risk for TB [27], and, in addition, injection drug use is a well-established risk factor for HIV and hepatitis C [33, 34].

Management of $\mathrm{TB}$ and alcohol use disorder comorbidity requires intensive patient support and social protection measures. Though this means additional resources, the cost-effectiveness of these interventions is likely to be high in view of potential to increase adherence to treatment regimens, prevent multidrug-resistant TB and mitigate socioeconomic effects and risk of further impoverishment associated with high societal costs. Health education, counselling, psychosocial and material support to patients are 
emphasised as important elements of TB case management in the new WHO TB treatment guidelines released in 2017 [35].

Finally, at population level, success can be achieved only if the variety of available public health approaches is effectively supported by coherent and linked policies and strategies aimed simultaneously at ending TB as a public health problem and reducing the harmful use of alcohol. To ensure this happens, the mounting evidence of the impact of the harmful use of alcohol on incidence and treatment outcomes of TB requires appropriate recognition at the level of strategic planning and political commitment backed by resources. The need for an integrated approach to care and prevention of complex public health problems like TB and harmful use of alcohol, built on strong health services and on societies as a whole is obvious. This approach is embedded in the 2030 Agenda for Sustainable Development where both ending $\mathrm{TB}$ and prevention and treatment of substance abuse are highlighted [36]. The strong causal relationship existing for noncommunicable conditions and TB, demanding synergistic, combined interventions, is also one of the key issues for discussion at the First WHO Global Ministerial Conference "Ending tuberculosis in the sustainable development era - a multisectoral response" to be held in Moscow on November 16-17, 2017. The expected Ministerial Declaration needs to emphasise the need for synergies and identify bold deliverables that can be subsequently put on the table of heads of state at the High Level Meeting on TB of the UN General Assembly in 2018. This will ensure commitment and willingness to finally respond adequately and reduce dramatically the burden of TB and the harmful use of alcohol that are ravaging humankind.

\section{References}

1 Lönnroth K, Raviglione M. Global epidemiology of tuberculosis: prospects for control. Semin Respir Crit Care Med 2008; 29: 481-491.

2 Lönnroth K, Castro GK, Chakaya JM, et al. Tuberculosis control and elimination 2010-50: cure, care, and social development. Lancet 2010; 375: 1814-1829.

3 Creswell J, Raviglione M, Ottmani S, et al. Tuberculosis and noncommunicable diseases: neglected links and missed opportunities. Eur Respir J 2011; 37: 1269-1282.

$4 \quad$ Omran AR. The Epidemiologic Transition. Milbank Mem Fund Q 1971; 49: 509-538.

5 GBD 2015 Mortality and Causes of Death Collaborators. Global, regional, and national life expectancy, all-cause mortality, and cause-specific mortality for 249 causes of death, 1980-2015: a systematic analysis for the Global Burden of Disease Study 2015. Lancet 2016; 388: 1459-1544.

6 World Health Organization. Global action plan for the prevention and control of noncommunicable diseases 2013-2020. Geneva, World Health Organization, 2013.

7 Rehm J, Baliunas D, Borges GLG, et al. The relation between different dimensions of alcohol consumption and burden of disease - an overview. Addiction 2010; 105: 817-843.

8 Parry J, Rehm J, Poznyak V, et al. Alcohol and infectious diseases: an overlooked causal linkage? Addiction 2009; 104: 331-332.

9 World Health Organization. Global strategy to reduce the harmful use of alcohol. Geneva, World Health Organization, 2010.

10 Rich AR. The patogenesis of tuberculosis. Baltimore, Charles C. Thomas, 1944.

11 Lönnroth K, Williams BG, Stadlin S, et al. Alcohol use as a risk factor for tuberculosis-a systematic review. BMC Public Health 2008; 8: 289.

12 Rehm J, Samokhvalov AV, Neuman MG, et al. The association between alcohol use, alcohol use disorders and tuberculosis (TB). A systematic review. BMC Public Health 2009; 9: 450.

13 World Health Organization. Global status report on alcohol and health 2014. Geneva, World Health Organization, 2014.

14 Forouzanfar MH, Alexander L, Anderson HR, et al. GBD 2013 Risk Factors Collaborators. Global, regional, and national comparative risk assessment of 79 behavioural, environmental and occupational, and metabolic risks or clusters of risks in 188 countries, 1990-2013: a systematic analysis for the Global Burden of Disease Study 2013. Lancet 2015; 386: 2287-2323.

15 Rehm J, Gmel GE Sr, Gmel G, et al. The relationship between different dimensions of alcohol use and the burden of disease - an update. Addiction 2017; 112: 968-1001.

16 Imtiaz S, Shield $\mathrm{KD}$, Roerecke $\mathrm{M}$, et al. Alcohol consumption as a risk factor for tuberculosis: meta-analyses and burden of disease. Eur Respir J 2017; 50: 1700216.

17 Dhanaraj B, Papanna MK, Adinarayanan S, et al. Prevalence and risk factors for adult pulmonary tuberculosis in a metropolitan city of South India. PloS One 2015; 10: e0124260.

18 Hermosilla S, You P, Aifah A, et al. Identifying risk factors associated with smear positivity of pulmonary tuberculosis in Kazakhstan. PloS One 2017; 12: e0172942.

19 Przybylski G, Dąbrowska A, Trzcińska H. Alcoholism and other socio-demographic risk factors for adverse TB-drug reactions and unsuccessful tuberculosis treatment-data from ten years' observation at the Regional Centre of Pulmonology, Bydgoszcz, Poland. Med Sci Monit 2014; 20: 444-453.

20 Vree M, Huong NT, Duong BD, et al. Mortality and failure among tuberculosis patients who did not complete treatment in Vietnam: a cohort study. BMC Public Health 2007; 7: 134.

21 Kendall EA, Theron D, Franke MF, et al. Alcohol, hospital discharge, and socioeconomic risk factors for default from multidrug resistant tuberculosis treatment in rural South Africa: a retrospective cohort study. PLoS One 2013; 8: e83480.

22 Peltzer K, Louw JS. Prevalence and associated factors of tuberculosis treatment outcome among hazardous or harmful alcohol users in public primary health care in South Africa. Afr Health Sci 2014; 14: 157-166. 
23 Kirenga BJ, Ssengooba W, Muwonge C, et al. Tuberculosis risk factors among tuberculosis patients in Kampala, Uganda: implications for tuberculosis control. BMC Public Health 2015; 15: 13.

24 Skrahina A, Hurevich H, Zalutskaya A, et al. Multidrug-resistant tuberculosis in Belarus: the size of the problem and associated risk factors. BullWorld Health Organ 2013; 91: 36-45.

25 Duraisamy K, Mrithyunjayan S, Ghosh S, et al. Does Alcohol consumption during multidrug-resistant tuberculosis treatment affect outcome? A population-based study in Kerala, India. Annals Am Thoracic Soc 2014; 11: 712-718.

26 Mulisa G, Workneh T, Hordofa N, et al. Multidrug-resistant Mycobacterium tuberculosis and associated risk factors in Oromia Region of Ethiopia. Int J Infect Dis 2015; 39: 57-61.

27 Smith CM, Trienekens SC, Anderson C, et al. Twenty years and counting: epidemiology of an outbreak of isoniazid-resistant tuberculosis in England and Wales, 1995 to 2014. Euro Surveill 2017; 22: 30467.

28 Viiklepp P, de Colombani P, Kurbatova A, et al. Collaborative action on tuberculosis and alcohol abuse in Estonia. First report of a demonstration project. World Health Organization, Regional Office for Europe, 2013.

29 Shin S, Livchits V, Connery HS, et al. Effectiveness of alcohol treatment interventions integrated into routine tuberculosis care in Tomsk, Russia. Addiction 2013; 108: 1387-1396.

30 World Health Organization. mhGAP intervention guide for mental, neurological and substance use disorders in non-specialized health settings: mental health Gap Action Programme (mhGAP) - version 2.0. Geneva, World Health Organization, 2016.

31 Jonas DE, Amick HR, Feltner C, et al. Pharmacotherapy for Adults With Alcohol Use Disorders in Outpatient Settings: A Systematic Review and Meta-analysis. JAMA 2014; 311: 1889-1900.

32 Connor JP, Haber PS, Hall WD. Alcohol use disorders. Lancet 2016; 387: 988-998.

33 World Health Organization. Global hepatitis report 2017. Geneva, World Health Organization, 2017.

34 The Joint United Nations Programme on HIV/AIDS (UNAIDS). Global AIDS update 2016. Geneva, UNAIDS, 2017.

35 World Health Organization. Guidelines for the treatment of drug-susceptible tuberculosis and patient care, 2017 update. WHO/HTM/TB/2017.05. Geneva, World Health Organization, 2017.

36 United Nations. United Nations General Assembly resolution 70/1. Transforming our world: the 2030 Agenda for Sustainable Development. United Nations, 2015. 